\title{
Testando o CAPM Condicional nos Mercados Brasileiro e Norte-Americano
}

\author{
Elmo Tambosi Filho \\ Newton C. A. da Costa Júnior \\ José Roberto Rossetto
}

\section{ResUmo}

Nas ultimas décadas o modelo CAPM tem despertado grande interesse por parte da comunidade científica. Apesar das críticas, o aprimoramento do CAPM estático, dando origem a novos modelos dinâmicos, traz maior segurança para o investidor ao longo do ciclo de negócios. O CAPM e suas versões estáticas foram e são de grande importância em finanças. Nos dias de hoje, encontramos adaptações mais complexas do modelo CAPM, as quais nos permitem ter respostas sobre questões em finanças que, por muito tempo, permaneceram não solucionadas. Diante deste panorama e considerando toda essa grande discussão acerca da validade do CAPM, este trabalho procura apresentar as vantagens dos modelos condicionais em relação ao modelo estático. Para constatar tais fatos estudar-se-ão os testes dos modelos condicionais (beta variando ao longo do tempo), que não são comumente estudados na literatura. Esses testes são convenientes para incorporar variâncias e covariâncias que se alteram ao longo do tempo. Dentre os testes dos modelos condicionais destacamos o de Jagannathan e Wang (1996). Conclui-se que esse modelo explica satisfatoriamente a variação cross-sectional dos retornos do mercado brasileiro e norte-americano.

Palavras-chave: CAPM condicional; mercados financeiros; portfolio.

\section{AbSTRACT}

In the last decades, the CAPM model has being of great interest in the scientific area. Despite all the criticism, the improvement of the static CAPM, which has generated new dynamic models, provided investors with stronger guarantee throughout the financial movement. The CAPM and its static version were and still are very important in the financial area. Nowadays more sophisticated adaptations of the CAPM are found, which allow us to explain some matters in finance that had not been solved for long time. Considering such discussion about the CAPM validity, this study intends to show the advantages of the conditional model comparing with the static one. In order to verify such facts, tests of conditional models are examined (with beta varying throughout time), which are not commonly studied in the literature. Such tests are suitable to incorporate variances and covariance that change throughout time. Among all, Jagannathan and Wang's (1996) can be considered one of the most important tests. This study aims to test the conditional CAPM model by Jagannathan and Wang (1996) using macroeconomics and financial variables from the Brazilian, Chilean and Argentinean markets. Also, one of its objectives is to compare such results with the American ones.

Key words: conditional CAPM; financial markets; portfolio. 


\section{INTRODUÇÃO}

Baseado nas idéias expostas por Markowitz, William Sharpe desenvolveu o Modelo do Índice Único, em 1963, que procurava simplificar a matriz de variâncias do modelo de Markowitz. Já em 1964 Sharpe publicou seu célebre artigo Capital Asset Prices: A Theory of Market Equilibrium under conditions of risk, estruturando, assim, o Capital Asset Pricing Model, um modelo para precificação dos ativos em mercados de títulos de risco em equilíbrio, o qual é amplamente utilizado e com importantes aplicações.

Em todos os testes do CAPM não condicional entre eles o de Fama e MacBeth (1973), Black, Jensen e Scholes (1972) foi suposto que o beta seria estático, ou seja, que o risco sistemático dos ativos não mudaria.

Nos estudos de Fama e MacBeth (1973), os betas de um período são usados para prever os retornos num período posterior. Fama e MacBeth (1973) mostram que nesses estudos empíricos do CAPM assumem que os betas permanecem constantes ao longo do tempo e o retorno dos portfolios, contendo as ações negociadas nos mercados, é uma boa proxy do retorno esperado do mercado.

Fama e French (1993) encontram em seu modelo de três fatores coeficientes estatisticamente significantes diferentes de zero. Este resultado sugere que a proxy dos fatores associados ao risco com o retorno do capital humano e o beta são instáveis. Mesmo assim, este modelo foi capaz de explicar a variação crosssectional dos retornos esperados.

O CAPM e suas versões estáticas foram e são de grande importância em finanças. Entretanto nos dias de hoje encontramos adaptações mais complexas do modelo CAPM que nos permitem vislumbrar respostas a questões que ainda permanecem sem resposta em finanças.

Diante deste panorama e considerando toda essa grande discussão acerca da validade do CAPM, este trabalho procura apresentar as vantagens dos modelos condicionais ou dinâmicos (modelos que incorporam variâncias e covariâncias que se alteram ao longo do tempo), em relação ao modelo estático.

Para constatar tais fatos estudar-se-ão os testes dos modelos condicionais (beta variando ao longo do tempo) que não são comumente estudados na literatura. Esses testes são convenientes para incorporar variâncias e covariâncias que se 
alteram ao longo do tempo. Dentre os testes dos modelos condicionais destacamos o de Jagannathan e Wang (1996) e Ferson e Harvey (1999).

Bonomo e Garcia (2002) destacam ainda, importantes estudos do CAPM condicional entre eles o proposto por Bodurtha e Mark (1991), onde o beta de uma carteira de ativos é definido como a covariância condicional do erro de previsão do retorno da carteira e o erro de previsão do retorno do mercado. Esses modelos possuem vários coeficientes betas enquanto o CAPM original possui apenas um.

Finalmente, esse trabalho encontra-se estruturado em cinco seções: a segunda seção traz algumas breves considerações sobre o CAPM Condicional. A terceira seção tecerá breves considerações sobre a metodologia de Fama e MacBeth (1973). A quarta seção traz alguns detalhes do modelo CAPM Condicional para o Brasil. A quinta seção mostra os resultados obtidos para o mercado brasileiro e norte-americano. E finalmente, a sexta seção mostra as conclusões finais.

\section{Algumas Considerações Iniciais acerca do CAPM Condicional}

O CAPM é definido como sendo um modelo que relaciona a rentabilidade esperada de um ativo ou bem, em um mercado em equilíbrio, com seu risco não diversificável, também conhecido pelo nome de beta. Independente de Sharpe, outros autores também formulam o CAPM, na sua versão estática. Entre eles Lintner (1965), Mossin (1966) e Treynor (1961). Esta versão do CAPM estático ou não condicional tem resultados consistentes ao se fazer testes empíricos para verificar a capacidade de aderência do modelo à economia real.

Em todos os testes do CAPM não condicional entre eles o de Fama e MacBeth (1973), Black, Jensen e Scholes (1972) foi suposto que o beta seria estático, ou seja, que o risco sistemático dos ativos não mudaria.

Haugen (1986) mostra que Black, Jensen e Scholes consideravam que deveria existir uma relação linear positiva entre os betas e as taxas de retorno esperado. Como conseqüência deste fato, Black et al. (1972) encontram em seu teste do CAPM um relacionamento positivo entre a rentabilidade e o beta.

Nos estudos de Fama e MacBeth (1973), os betas de um período são usados para prever os retornos num período posterior. Fama e MacBeth (1973) mostram que nesses estudos empíricos do CAPM assumem que os betas permanecem constantes ao longo do tempo e o retorno dos portfolios contendo as ações negociadas nos mercados é uma boa proxy do retorno esperado do mercado. 
Merton (1973) mostra que o Consumption Capital Asset Pricing Model (ICAPM) tinha como objetivo generalizar o modelo CAPM de Sharpe (1964) para um contexto intertemporal. O ICAPM original parte da hipótese de que os investidores consumiam toda a sua riqueza depois de um período, de forma que a riqueza e o consumo acabavam se confundindo.

Já o CAPM estático de Sharpe-Lintner-Black, dado por $R_{i}$ que denota o retorno da ação ${ }_{i}$ e $R_{m}$ o retorno do portfolio de mercado para todas as ações da economia. A versão do CAPM de Black (1972) é:

$E\left[R_{i}\right]=\gamma_{0}+\gamma_{1} \beta_{i}$

onde $\gamma_{0}$ e $\gamma_{1}$ são definidos como o retorno esperado de mercado e o prêmio do risco esperado de mercado respectivamente e onde $\beta_{i}$ é definido como:

$\beta_{i}=\operatorname{Cov}\left(R_{i}, R_{m}\right) / \operatorname{Var}\left[R_{m}\right]$

Fama e French (1992) seguem Black (1972) e examinam empiricamente o CAPM estático, chegando à conclusão que existe uma relação fraca entre o retorno médio e o beta, encontrando uma forte evidência contra o CAPM estático.

Entretanto, Jagannathan e Wang (1996) desenvolveram um estudo que em parte contrariavam essas evidências.

Nesse mesmo estudo observaram que, ao utilizar o índice CRSP como portfolio de mercado, encontram no modelo não-condicional, implícito no CAPM condicional, uma explicação aproximada de $30 \%$ da variação cross-sectional do retorno médio de 100 portfolios de mercado, similar ao utilizado por Fama e French (1992). Para implementação do CAPM é comumente utilizado como proxy todos as ações listadas na New York Stock Exchange (NYSE) e na American Stock Exchange (AMEX), que podem ser consideradas uma razoável proxy para o retorno do portfolio de mercado de todos os ativos.Contudo, Fama e French (1992) constataram que, ao utilizar tal proxy, a mesma não foi suficiente para uma análise satisfatória da performance do CAPM.

Devido a esse fato e com o objetivo de melhorar a proxy, Jagannathan e Wang (1996) seguiram Mayers (1972) e incluíram em seu modelo o retorno do capital humano. Quando o capital humano é também incluído na carteira de mercado, o modelo não-condicional implícito no CAPM condicional é capaz de explicar mais de $50 \%$ da variação cross-sectional do retorno médio. Além disso, os testes estatísticos falharam na rejeição do modelo. 


\section{A Metodologia de Fama e MacBeth (1973)}

Haugen (1986) mostra que a metodologia de Fama e MacBeth (1973) introduziu diferenças significativas em relação aos testes anteriores, já que chegaram a resultados coerentes com as previsões fundamentais do CAPM (versão de Black, 1972).

Fama e MacBeth (1973) formaram 20 portfolios contendo ações listadas na NYSE do período de 1926 até 1929. Posteriormente eles estimaram o beta de cada um dos portfolios relacionando os retornos mensais do índice de mercado para o período de 1930 até 1934. Utilizaram os betas de cada um dos portfolios do período anterior para prever os retornos mensais dos portfolios do período subseqüente de 1935 até 1938 . O processo de estimação dos betas do mercado se repetiu nove vezes até a obtenção total de 390 estimações que foi de janeiro de 1935 até junho de 1968.

Fama e MacBeth utilizaram o valor médio de cada um dos coeficientes para determinar a sua significância. Concluiu-se que portfolios com betas maiores do que a média tenderão a produzir taxas de retorno maiores que a média nos períodos subseqüentes. Existe pouca ou nenhuma evidência da não linearidade na relação entre o beta e o retorno. Também, eles verificaram que não se pode prever o retorno futuro com base na variância residual dos ativos do portfolio. Em relação ao CAPM, não se pode esperar que a variância residual afete o preço dos ativos ou a taxa de retorno esperada e, baseado nos resultados mostrados pelos autores, não existe uma indicação de que os ativos com variância residual maior que a média produzam taxa de retorno maior que a média num período futuro.

Ressalta-se que foram selecionadas variáveis que representam, de forma mais real, a situação macroeconômica no Brasil. Para isso foi substituída a variável labor pela variável PIB do mercado mensal (Brasil), e a variável prêmio pelo spread entre as taxas de operações de depósitos interfinanceiros (CDI) da CETIP e a taxa de juros Selic para o mercado brasileiro. O spread entre o CDI e a taxa de juros Selic foi utilizado por representar, de maneira mais realista, a relação entre os papéis do setor privado e do setor público no Brasil.

Jagannathan e Wang (1996) utilizaram critérios semelhantes para a escolha dessas variáveis procurando dar mais realismo ao modelo.

Haugen (1986) mostra que, neste caso, Fama e MacBeth utilizaram betas e retornos em diferentes períodos. Os betas estimados em um período são usados para prever as taxas de retorno para o período posterior. Os resultados destes 
testes foram muito confortantes e, de fato, o CAPM ganhou uma forte aceitação entre os acadêmicos após a publicação do estudo.

Apesar das críticas a esse modelo, encontramos ainda muitos estudos que se baseiam nele. O que pode ser visto a seguir através do modelo de Jagannathan e Wang (1996) que usa a mesma metodologia de Fama e MacBeth (1973).

\section{O Modelo CAPM Condicional para o Brasil}

As variáveis selecionadas a priori que farão parte do modelo CAPM condicional para o Brasil, referem-se a portfolios construídos por meio dos retornos mensais de ações cotadas na Bolsa de Valores de São Paulo (Bovespa), PIB de mercado e, para o prêmio, o spread entre a taxa de operações de depósitos interfinanceiros (DI), divulgadas pela Central de Custódia e Liquidação de Títulos Privados (CETIP) e a taxa de juros (Selic), que tem a finalidade de ser um previsor para as variações dos ciclos de negócios.

O período escolhido foi de janeiro 1994 a dezembro 2002, totalizando 108 observações. Este período foi escolhido devido à maior estabilidade das variáveis macroeconômicas no mercado brasileiro quando comparado com outros períodos. Os dados foram coletados no Banco Central do Brasil e Economática. Foram utilizadas trinta e cinco ações divididas em sete portfolios para o mercado brasileiro.

Seguindo os passos acima, Jagannathan e Wang (1996), utilizaram os retornos de todas ações da NYSE e AMEX e montaram 100 portfolios em função da variável tamanho, com retornos mensais de julho de 1963 a dezembro de 1990, totalizando 330 observações. Para cada portfolio calculam-se regressões entre as ações que compõem o portfolio e o índice de mercado (NYSE e AMEX).

As regressões são estimadas usando a metodologia de Fama e MacBeth (1973). Elaboraram-se 7 portfolios contendo ações listadas na Bovespa que foram analisadas no período de 1994 até 2002. Posteriormente estimamos o beta de cada um dos portfolios, relacionando os retornos mensais do índice de mercado para o período de 1994 até 1998. Utilizamos os betas de cada um dos portfolios do período anterior para prever os retornos mensais dos portfolios do período subsequiente de 1999 até 2002. O processo de estimação dos betas do mercado se repetiu seis vezes até a obtenção total de 108 estimações que foi de janeiro de 1994 até dezembro de 2002. Ainda, em relação ao beta, foi estimado para cada ação, usando 24 e 60 meses do retorno passado das ações das bolsas analisadas. Seguindo Fama e French (1992), foram criados 7 portfolios, e computou-se o 
retorno de cada um deles para os 12 meses seguintes. Foi repetido esse procedimento para cada ano analisado.

Criou-se uma série temporal do retorno mensal para cada um dos 7 portfolios. O modelo para os momentos é estimado usando o método dos momentos generalizado. Utilizou-se o valor médio de cada um dos coeficientes para determinar a sua significância. Os portfolios foram sendo rebalanceados anualmente.

De acordo com Fama e MacBeth (1973) esses portfolios eram rebalanceados período por período, até que a estimação dos betas atingisse o total de estimações de todo o período analisado. Todas as ações receberam o mesmo peso dentro de cada portfolio.

Já a escolha das ações se deu levando em conta o critério da liquidez e utilizando apenas ações sobreviventes, isto é, ações de empresas que permaneceram cotadas na Bolsa de Valores de São Paulo durante todo o período de análise do estudo. Fama e French (1988) mostram que essa construção, apesar de arbitrária, é comum na literatura de testes empíricos. Apesar de Fama e French (1992) e Jagannathan e Wang (1996) não usarem ações de bancos em suas amostras, optou-se pelo uso destas ações, porque testes de versões do modelo CAPM no Brasil já utilizam essas ações (Ribenboim, 2002).

\section{Resultados Obtidos para o Mercado Brasileiro e Norte- AMERICANO}

O aprimoramento do CAPM estático, dando origem a novos modelos dinâmicos, traz maior segurança para o investidor ao longo do ciclo de negócios. O CAPM mostra que o risco de um ativo financeiro é bem representado pelo coeficiente beta que mede a adequação de um título ao mercado.

Os resultados presentes nas Tabelas 1 e 2 mostram que o $t$ value para o $C_{\text {ibov }}$ é 0,17 . O R ${ }^{2}$ da regressão é de somente $8,49 \%$. Isto quer dizer que a variação cross-sectional média dos retornos ainda não é bem explicada, quando utilizamos o CAPM estático sem a inclusão do PIB de mercado no caso brasileiro. Essa análise evidencia a forte relação das variáveis macroeconômicas, no caso o PIB, que não é contemplado nesta primeira tabela. 


\section{Tabela 1: CAPM Estático Sem Capital Humano (Brasil)}

\begin{tabular}{lllllll}
\hline Coeficientes: & $\mathrm{C}_{0}$ & $\mathrm{C}_{\text {ibov }}$ & $\mathrm{C}_{\text {premio }}$ & $\mathrm{C}_{\text {pib mer }}$ & $\mathrm{C}_{\text {size }}$ & R-square \\
\hline Estimate: & $-2,67$ & 1,25 & & & & 8,49 \\
t-value: & $-0,76$ & 0,17 & & & & \\
p-value: & 0,00 & 0,00 & & & & \\
Correção -t: & $-0,19$ & 0,08 & & & \\
Correção- $\mathrm{p}:$ & 0,00 & 0,00 & & & \\
Estimate: & $-0,88$ & 0,44 & & 0,61 & 42,10 \\
t-value: & $-0,24$ & 0,06 & & 3,29 & \\
p-value: & 0,00 & 0,00 & & 0,00 & \\
Correção -t: & $-0,14$ & 0,05 & & 2,97 & \\
Correção -p: & 0,00 & 0,00 & & 0,00 & \\
\hline
\end{tabular}

As regressões do modelo são estimadas usando a metodologia de Fama e MacBeth (1973). O modelo foi estimado usando o método generalizado dos momentos. Por meio da correção dos erros, verificamos se a variância residual afeta o preço dos ativos ou a taxa de retorno esperada e, baseado nos resultados, não existe indicação de que os ativos com variância residual maior que a média produzam taxa de retorno maior que a média num período futuro. Foram construídos sete portfolios com cinco ações em cada um deles. O período testado vai de janeiro de 1994 até dezembro de 2002. Para o mercado brasileiro o prêmio é representado pelo spread entre a taxa da CETIP e a taxa SELIC. Já o capital humano é representado pelo PIB de mercado. A proxy de mercado será o Ibovespa. A equação que está sendo estimada para o mercado brasileiro é a seguinte: $E\left[R_{i t}\right]=c_{0}+c_{\text {size }} \log \left(M E_{i}\right)+c_{\text {ibov }} \beta+c_{\text {premio }} \beta+c_{\text {pib.mer }} \beta$

Tabela 2: CAPM Estático Sem Capital Humano (EUA)

\begin{tabular}{|c|c|c|c|c|c|c|}
\hline Coeficientes: & $\mathrm{C}_{0}$ & $\mathrm{C}_{\mathrm{vw}}$ & $\mathrm{C}_{\text {prem }}$ & $\mathrm{C}_{\text {labor }}$ & $\mathrm{C}_{\text {size }}$ & $\mathrm{R}$-square \\
\hline Estimate: & 1.24 & -0.10 & & & & 1.35 \\
\hline t-value: & 5.17 & -0.28 & & & & \\
\hline p-value: & 0.00 & 78.00 & & & & \\
\hline Corrected-t: & 5.16 & -0.28 & & & & \\
\hline Corrected-p: & 0.00 & 78.01 & & & & \\
\hline Estimate: & 2.08 & -0.32 & & & -0.11 & 57.56 \\
\hline t-value: & 5.79 & -0.94 & & & -2.30 & \\
\hline p-value: & 0.00 & 34.54 & & & 2.14 & \\
\hline Corrected-t: & 5.77 & -0.94 & & & -2.30 & \\
\hline Corrected-p: & 0.00 & 34.60 & & & 2.17 & \\
\hline
\end{tabular}

As regressões do modelo são estimadas usando a metodologia de Fama e MacBeth (1973). O modelo foi estimado usando o método generalizado dos momentos. Por meio da correção dos erros, verificamos se a variância residual afeta o preço dos ativos ou a taxa de retorno esperada e, baseado nos resultados, não existe indicação de que os ativos com variância residual maior que a média produzam taxa de retorno maior que a média num período futuro. Foram construídos sete portfolios com cinco ações em cada um deles. O período testado vai de janeiro de 1994 até dezembro de 2002. Para o mercado brasileiro o prêmio é representado pelo spread entre a taxa da CETIP e a taxa SELIC. Já o capital humano é representado pelo PIB de mercado. A proxy de mercado será o Ibovespa. A equação que está sendo estimada para o mercado brasileiro é a seguinte: $E\left[R_{i t}\right]=c_{0}+c_{\text {size }} \log \left(M E_{i}\right)+c_{\text {ibov }} \beta+c_{\text {premio }} \beta+c_{\text {pib.mer }} \beta$ 
O modelo de correção de erros para estimação não é significativo. Após a correção dos erros, que trata o termo de erro do modelo, podendo usar este termo para ligar o comportamento das variáveis no curto prazo com seu valor no longo prazo, ou seja, é um meio de reconciliar o comportamento no curto prazo de uma variável com seu comportamento no longo prazo. Conclui-se, então, que o $\mathrm{C}_{\mathrm{ibov}}$ não é significativamente diferente de zero. Quando é introduzida a variável size no modelo, encontramos para o $\mathrm{C}_{\text {size }}$ um $t$-value de 3,29 e o $\mathrm{R}^{2}$ sobe para $42,10 \%$. A variável size para o modelo brasileiro parece ter uma influência menor do que no modelo norte-americano.

Apesar do aumento do $\mathrm{R}^{2}$ e do fato de o modelo não apresentar nenhuma mudança significativa após a correção dos erros, o modelo parece inconsistente, porque, mesmo após a inclusão da variável size, para o mercado brasileiro este parece não ser influenciado por esta, devido a que o modelo estático não capta $\mathrm{o}$ efeito dessa variável. A análise do mercado brasileiro parece caminhar para uma conclusão semelhante àquela do mercado norte-americano.

Os resultados presentes nas Tabelas 3 e 4 mostram que o valor estimado para o $\mathrm{C}_{\text {premio }}$, não é significativamente diferente de zero. $\mathrm{O} t$-value para $\mathrm{C}_{\text {premio }}$ é de 0,52 . O R $\mathrm{R}^{2}$ é de somente $9,82 \%$. Nota-se que o $\mathrm{R}^{2}$ é similar ao resultado encontrado no modelo anterior.

Tabela 3: CAPM Condicional Sem Capital Humano (Brasil)

\begin{tabular}{lllllll}
\hline Coeficientes: & $\mathrm{C}_{0}$ & $\mathrm{C}_{\mathrm{ibov}}$ & $\mathrm{C}_{\text {premio }}$ & $\mathrm{C}_{\text {pib mer }}$ & $\mathrm{C}_{\text {size }}$ & R-square \\
\hline Estimate: & $-2,67$ & $-1,09$ & $-4,74$ & & & 9,82 \\
t-value: & $-0,74$ & $-0,05$ & $-0,52$ & & & \\
p-value: & 0,00 & 0,00 & 0,00 & & & \\
Correção -t: & $-0,17$ & $-0,02$ & $-0,36$ & & & \\
Correção -p: & 0,00 & 0,01 & 0,00 & & & \\
Estimate: & $-0,87$ & 0,56 & 0,53 & & 0,61 & 42,90 \\
t-value: & $-0,20$ & 0,02 & 0,33 & 3,03 & \\
p-value: & 0,00 & 0,08 & 0,32 & 0,00 & \\
Correção-t: & $-0,14$ & 0,01 & 0,16 & & 2,68 & \\
Correção-p: & 0,00 & 0,05 & 0,51 & & 0,00 & \\
\hline As & & & & & & \\
\hline
\end{tabular}

As regressões do modelo são estimadas usando a metodologia de Fama e MacBeth (1973). O modelo foi estimado usando o método generalizado dos momentos. Por meio da correção dos erros verificamos se a variância residual afeta o preço dos ativos ou a taxa de retorno esperada e, baseado nos resultados, não existe indicação de que os ativos com variância residual maior que a média produzam taxa de retorno maior que a média num período futuro. Foram construídos sete portfolios com cinco ações em cada um deles. O período testado vai de janeiro de 1994 até dezembro de 2002. Para o mercado brasileiro o prêmio é representado pelo spread entre a taxa da CETIP e a taxa SELIC. Já o capital humano é representado pelo PIB de mercado. A proxy de mercado será o Ibovespa. A equação que está sendo estimada para o mercado brasileiro é a seguinte: $E\left[R_{i t}\right]=c_{0}+c_{\text {size }} \log \left(M E_{i}\right)+c_{i b o v} \beta+c_{\text {premio }} \beta+c_{\text {pib.mer }} \beta$ 
Tabela 4: CAPM Condicional Sem Capital Humano (EUA)

\begin{tabular}{lllllll}
\hline Coeficientes: & $\mathrm{C}_{0}$ & $\mathrm{C}_{\mathrm{vw}}$ & $\mathrm{C}_{\text {prem }}$ & $\mathrm{C}_{\text {labor }}$ & $\mathrm{C}_{\text {size }}$ & R-square \\
\hline Estimate: & 0.81 & -0.31 & 0.36 & & & 29.32 \\
t-value: & 2.72 & -0.87 & 3.28 & & & \\
p-value: & 0.66 & 38.45 & 0.10 & & & \\
Corrected-t: & 2.19 & -0.70 & 2.67 & & & \\
Corrected-p: & 2.87 & 48.43 & 0.75 & & & \\
Estimate: & 1.77 & -0.38 & 0.16 & -0.10 & 61.66 \\
t-value: & 4.75 & -1.10 & 2.50 & -1.93 & \\
p-value: & 0.00 & 27.17 & 1.26 & 5.35 & \\
Corrected-t: & 4.53 & -1.05 & 2.40 & & -1.84 & \\
Corrected-p: & 0.00 & 29.53 & 1.66 & & 6.59 & \\
\hline A regress & & & & & &
\end{tabular}

As regressões do modelo são estimadas usando a metodologia de Fama e MacBeth (1973). O modelo foi estimado usando o método generalizado dos momentos. Por meio da correção dos erros verificamos se a variância residual afeta o preço dos ativos ou a taxa de retorno esperada e, baseado nos resultados, não existe indicação de que os ativos com variância residual maior que a média produzam taxa de retorno maior que a média num período futuro. Foram construídos sete portfolios com cinco ações em cada um deles. O período testado vai de janeiro de 1994 até dezembro de 2002. Para o mercado brasileiro o prêmio é representado pelo spread entre a taxa da CETIP e a taxa SELIC. Já o capital humano é representado pelo PIB de mercado. A proxy de mercado será o Ibovespa. A equação que está sendo estimada para o mercado brasileiro é a seguinte: $E\left[R_{i t}\right]=c_{0}+c_{\text {size }} \log \left(M E_{i}\right)+c_{i b o v} \beta+c_{\text {premio }} \beta+c_{\text {pib.mer }} \beta$

Quando se introduz o modelo de correção de erros, o $t$-value para $\mathrm{C}_{\text {premio }}$ é de - 0,36. Quando a variável size é adicionada ao modelo o t-value para $\mathrm{C}_{\text {size }}$ é de 3,03. Quando se introduz o modelo de correção de erros, o $t$-value para o $\mathrm{C}_{\text {size }}$ cai para 2,68 , e o $\mathrm{R}^{2}$ sobe para $42,90 \%$.

Verifica-se que o valor do $\mathrm{R}^{2}$ se manteve em $42,90 \%$ (valor muito parecido com o encontrado no CAPM estático) e o valor estimado para a variável $\mathrm{C}_{\text {premio }}$, depois de corrigidos os erros, se tornou significativamente diferente de zero, fatos estes que podem ser explicados pela não inclusão do PIB de mercado.

Assim mesmo, o modelo condicional parece ser mais eficaz para explicar a variação cross-sectional média dos retornos no mercado brasileiro. Análise semelhante ao mercado norte-americano.

O modelo condicional proposto por Jaganathan e Wang (1996) parece ser mais eficiente nas previsões, mesmo quando não incluímos o PIB de mercado.

Bonomo e Garcia (2002) mostram que o CAPM condicional tem maior aderência aos dados, capturando melhor a dinâmica das medidas de risco e dos retornos esperados. A utilização do CAPM condicional no mercado de ações brasileiro e norte-americano deverá proporcionar mais um instrumento de gerenciamento de carteiras de ativos. 
Os resultados presentes nas Tabelas 5 e 6 mostram que o valor estimado para o $\mathrm{C}_{\text {pibmer }}$, usando a metodologia de Fama-MacBeth, não é significativamente diferente de zero. O t-value é de - 0,21 e o $\mathrm{R}^{2}$ é de $11,76 \%$.

Tabela 5: CAPM Condicional com Capital Humano (Brasil)

\begin{tabular}{lllllll}
\hline Coeficientes: & $\mathrm{C}_{0}$ & $\mathrm{C}_{\mathrm{ibov}}$ & $\mathrm{C}_{\text {premio }}$ & $\mathrm{C}_{\text {pib mer }}$ & $\mathrm{C}_{\text {size }}$ & R-square \\
\hline Estimate: & $-3,01$ & 4,67 & 3,89 & $-0,59$ & & 11,76 \\
t-value: & $-0,78$ & 0,12 & $-0,16$ & $-0,21$ & & \\
p-value: & 0,00 & 0,00 & 0,00 & 0,00 & & \\
Correção -t: & $-0,1$ & 0,01 & $-0,09$ & $-0,03$ & & \\
Correção-p: & 0,00 & 0,04 & 0,10 & 0,02 & & \\
Estimate: & $-0,85$ & 13,25 & 2,86 & $-1,28$ & 0,69 & 51,59 \\
t-value: & $-0,2$ & 0,33 & 0,18 & $-0,44$ & 2,62 & \\
p-value: & 0,00 & 0,00 & 0,00 & 0,00 & 0,00 & \\
Correção-t: & $-0,01$ & 0,02 & 0,10 & $-0,03$ & 0,21 & \\
Correção-p: & 0,04 & 0,02 & 0,85 & 0,01 & 0,00 & \\
\hline
\end{tabular}

As regressões do modelo são estimadas usando a metodologia de Fama e MacBeth (1973). O modelo foi estimado usando o método generalizado dos momentos. Por meio da correção dos erros, verificamos se a variância residual afeta o preço dos ativos ou a taxa de retorno esperada e, baseado nos resultados, não existe indicação de que os ativos com variância residual maior que a média produzam taxa de retorno maior que a média num período futuro. Foram construídos sete portfolios com cinco ações em cada um deles. O período testado vai de janeiro de 1994 até dezembro de 2002. Para o mercado brasileiro o prêmio é representado pelo spread entre a taxa da CETIP e a taxa SELIC. Já o capital humano é representado pelo PIB de mercado. A proxy de mercado será o Ibovespa. A equação que está sendo estimada para o mercado brasileiro é a seguinte: $E\left[R_{i t}\right]=c_{0}+c_{\text {size }} \log \left(M E_{i}\right)+c_{\text {ibov }} \beta+c_{\text {premio }} \beta+c_{\text {pib.mer }} \beta$

\section{Tabela 6: CAPM Condicional com Capital Humano (EUA)}

\begin{tabular}{lllllll}
\hline Coeficientes: & $\mathrm{C}_{0}$ & $\mathrm{C}_{\mathrm{vw}}$ & $\mathrm{C}_{\text {prem }}$ & $\mathrm{C}_{\text {labor }}$ & $\mathrm{C}_{\text {size }}$ & $\mathrm{R}$-square \\
\hline Estimate: & 1.24 & -0.40 & 0.34 & 0.22 & & 55.21 \\
t-value: & 5.51 & -1.18 & 3.31 & 2.31 & & \\
p-value: & 0.00 & 23.76 & 0.09 & 2.07 & & \\
Corrected-t: & 4.10 & -0.88 & 2.48 & 1.73 & & \\
Corrected-p: & 0.00 & 37.99 & 1.31 & 8.44 & & \\
Estimate: & 1.70 & -0.40 & 0.20 & 0.10 & -0.07 & 64.73 \\
t-value: & 4.61 & -1.18 & 3.00 & 2.09 & -1.45 & \\
p-value: & 0.00 & 23.98 & 0.27 & 3.62 & 14.74 & \\
Corrected-t: & 4.14 & -1.06 & 2.72 & 1.89 & -1.30 & \\
Corrected-p: & 0.00 & 29.07 & 0.66 & 5.87 & 19.29 & \\
\hline
\end{tabular}

As regressões do modelo são estimadas usando a metodologia de Fama e MacBeth (1973). O modelo foi estimado usando o método generalizado dos momentos. Por meio da correção dos erros, verificamos se a variância residual afeta o preço dos ativos ou a taxa de retorno esperada e, baseado nos resultados, não existe indicação de que os ativos com variância residual maior que a 
média produzam taxa de retorno maior que a média num período futuro. Foram construídos sete portfolios com cinco ações em cada um deles. O período testado vai de janeiro de 1994 até dezembro de 2002. Para o mercado brasileiro o prêmio é representado pelo spread entre a taxa da CETIP e a taxa SELIC. Já o capital humano é representado pelo PIB de mercado. A proxy de mercado será o Ibovespa. A equação que está sendo estimada para o mercado brasileiro é a seguinte: $E\left[R_{i t}\right]=c_{0}+c_{\text {size }} \log \left(M E_{i}\right)+c_{\text {ibov }} \beta+c_{\text {premio }} \beta+c_{\text {pib.mer }} \beta$

Entretanto, quando se introduz o modelo de correção de erros, o $t$-value para $\mathrm{C}_{\text {pibmerr }}$ cai para -0,03, o $p$-value vai para 0,02 e o coeficiente $\mathrm{C}_{\text {premio }}$ torna-se significante. Quando a variável size é adicionada ao modelo, o $t$-value para o $\mathrm{C}_{\text {size }}$ é de 2,62,e o $\mathrm{R}^{2}$ sobe para $51,59 \%$.

Conclui-se que o CAPM condicional com a inclusão do PIB de mercado brasileiro parece aproximar-se dos resultados obtidos no mercado norte-americano. Concluise ainda que, apesar de as variáveis $\mathrm{C}_{\text {premio }}$ e $\mathrm{C}_{\text {pib.mer }}$ se tornarem significativamente diferentes de zero após a correção dos erros, a consistência do modelo não parece ter sido abalada.

Entretanto a performance substancialmente melhor do modelo condicional requer alguns cuidados na sua interpretação. Um importante aspecto, que devemos levar em conta, diz respeito ao tamanho da amostra utilizada. Segundo Ribenboim (2002), a literatura existente sobre os testes do modelo CAPM indica que o ideal é ter uma amostra que nos permita algumas subdivisões em períodos de dez anos.

Os resultados presentes nas Tabelas 7 e 8 mostram que o valor estimado para o $\mathrm{C}_{\text {pib.merr }}$, usando a metodologia de Fama-MacBeth, não é significativamente diferente de zero. O $t$-value é de $-0,23$. O $\mathrm{R}^{2}$ é de apenas $10,75 \%$. Após a correção dos erros, conclui-se que o $\mathrm{C}_{\text {lpib.mer }}$ se torna significativamente diferente de zero, ao contrário do que ocorre no mercado norte-americano.

Tabela 7: CAPM Estático com Capital Humano (Brasil)

\begin{tabular}{lllllll}
\hline Coeficientes: & $\mathrm{C}_{0}$ & $\mathrm{C}_{\mathrm{ibov}}$ & $\mathrm{C}_{\text {premio }}$ & $\mathrm{C}_{\text {pib mer }}$ & $\mathrm{C}_{\text {size }}$ & R-square \\
\hline Estimate: & $-2,83$ & 6,91 & & $-0,62$ & & 10,75 \\
t-value: & $-0,79$ & 0,30 & & $-0,23$ & & \\
p-value: & 0,00 & 0,00 & & 0,00 & & \\
Correção-t: & $-0,09$ & 0,03 & & $-0,03$ & & \\
Correção-p: & 0,00 & 0,01 & & 0,04 & & \\
Estimate: & $-0,98$ & 11,78 & $-1,25$ & 0,68 & 50,83 \\
t-value: & $-0,26$ & 0,49 & $-0,46$ & 3,29 & \\
p-value: & 0,00 & 0,00 & 0,00 & 0,00 & \\
Correção-t: & $-0,02$ & 0,03 & $-0,03$ & 0,28 & \\
Correção-p: & 0,02 & 0,01 & & 0,05 & 0,00 & \\
\hline
\end{tabular}


As regressões do modelo são estimadas usando a metodologia de Fama e MacBeth (1973). O modelo foi estimado usando o método generalizado dos momentos. Por meio da correção dos erros, verificamos se a variância residual afeta o preço dos ativos ou a taxa de retorno esperada e, baseado nos resultados, não existe indicação de que os ativos com variância residual maior que a média produzam taxa de retorno maior que a média num período futuro. Foram construídos sete portfolios com cinco ações em cada um deles. O período testado vai de janeiro de 1994 até dezembro de 2002. Para o mercado brasileiro o prêmio é representado pelo spread entre a taxa da CETIP e a taxa SELIC. Já o capital humano é representado pelo PIB de mercado. A proxy de mercado será o Ibovespa. A equação que está sendo estimada para o mercado brasileiro é a seguinte: $E\left[R_{i t}\right]=c_{0}+c_{\text {size }} \log \left(M E_{i}\right)+c_{\text {ibov }} \beta+c_{\text {premio }} \beta+c_{\text {pib.mer }} \beta$

\section{Tabela 8: CAPM Estático com Capital Humano (EUA)}

\begin{tabular}{|c|c|c|c|c|c|c|}
\hline Coeficientes: & $\mathrm{C}_{0}$ & $\mathrm{C}_{\mathrm{vw}}$ & $\mathrm{C}_{\text {prem }}$ & $\mathrm{C}_{\text {labor }}$ & $\mathrm{C}_{\text {size }}$ & R-square \\
\hline Estimate: & 1.67 & -0.22 & & 0.23 & & 30.46 \\
\hline t-value: & 6.91 & -0.63 & & 2.37 & & \\
\hline p-value: & 0.00 & 53.19 & & 1.77 & & \\
\hline Corrected-t: & 5.71 & -0.52 & & 1.97 & & \\
\hline Corrected-p: & 0.00 & 60.49 & & 4.87 & & \\
\hline Estimate: & 2.09 & -0.32 & & 0.05 & -0.10 & 58.55 \\
\hline t-value: & 5.80 & -0.96 & & 1.22 & -2.15 & \\
\hline p-value: & 0.00 & 33.78 & & 22.29 & 3.19 & \\
\hline Corrected-t: & 5.70 & -0.95 & & 1.20 & -2.11 & \\
\hline Corrected-p: & 0.00 & 34.46 & & 22.93 & 3.48 & \\
\hline
\end{tabular}

As regressões do modelo são estimadas usando a metodologia de Fama e MacBeth (1973). O modelo foi estimado usando o método generalizado dos momentos. Por meio da correção dos erros, verificamos se a variância residual afeta o preço dos ativos ou a taxa de retorno esperada e, baseado nos resultados, não existe indicação de que os ativos com variância residual maior que a média produzam taxa de retorno maior que a média num período futuro. Foram construídos sete portfolios com cinco ações em cada um deles. O período testado vai de janeiro de 1994 até dezembro de 2002. Para o mercado brasileiro o prêmio é representado pelo spread entre a taxa da CETIP e a taxa SELIC. Já o capital humano é representado pelo PIB de mercado. A proxy de mercado será o Ibovespa. A equação que está sendo estimada para o mercado brasileiro é a seguinte: $E\left[R_{i t}\right]=c_{0}+c_{\text {size }} \log \left(M E_{i}\right)+c_{i b o v} \beta+c_{\text {premio }} \beta+c_{\text {pib.mer }} \beta$

Quando introduzirmos a variável size, o t-value é de 3,29 e o $\mathrm{R}^{2}$ sobe para $50,83 \%$. Apesar do elevado $\mathrm{R}^{2}$, o modelo não é consistente. É necessário permitir que o beta varie no tempo para explicar o retorno cross-sectional esperado dos mercados.

Os resultados empíricos do modelo CAPM condicional, com dados da economia americana, nos permitiram contemplar um campo para testes similares com série de dados do mercado brasileiro. 


\section{Conclusões Finais}

Verifica-se que o CAPM estático, sem a inclusão da variável capital humano, parece não explicar satisfatoriamente o retorno cross-sectional esperado dos mercados analisados.

Após a inclusão da variável size, o $\mathrm{R}^{2}$ de todos modelos tem uma abrupta mudança. Apesar deste fato e de que os resultados encontrados são coerentes com a literatura, conclui-se que o modelo para os países analisados parece inconsistente por não apresentar nenhuma mudança nos parâmetros ao longo do tempo.

Este modelo parece não representar satisfatoriamente a realidade de cada economia. Primeiramente, porque sabemos que o ciclo de negócios é dinâmico em qualquer economia e o modelo analisado acima não contempla este fato. Segundo, porque a proxy de mercado, por si só, não é suficiente para representar uma economia, seja ela qual for.

Conclui-se, então, que este modelo deve ser aperfeiçoado com a inclusão de novas variáveis que consiga representar bem qualquer que seja o mercado.

Entretanto não devemos desprezar o CAPM estático, pois ele é capaz de explicar o mercado para um determinado espaço de tempo.

Em relação ao CAPM condicional, sem a inclusão da variável capital humano, podemos constatar que, no caso brasileiro, o valor estimado para o $\mathrm{C}_{\text {premio }}$ não é significativamente diferente de zero, enquanto no caso norte-americano é significativamente diferente de zero.

Entretanto, quando se introduz o modelo de correção dos erros, a variável $\mathrm{C}_{\text {premio }}$ torna-se significativamente diferente de zero para o caso brasileiro. No caso norte-americano, mesmo após a adoção do modelo de correção de erros, a variável $\mathrm{C}_{\text {premio }}$ se mantém significativamente diferente de zero. Isto significa que o prêmio de risco influencia demasiadamente o mercado analisado.

Quando a variável size é adicionada ao modelo, o $\mathrm{R}^{2}$ sobe proporcionalmente para os dados brasileiros. Para os dados norte-americanos, quando é incluída a variável size, o $\mathrm{R}^{2}$ sobe de forma menos significativa.

Conclui-se que, quando a variável size é adicionada aos modelos, seus $\mathrm{R}^{2}$ sofrem aumento considerável, apesar de a variável size apresentar alguns efeitos no modelo. Isto quer dizer que o CAPM condicional, mesmo sem a inclusão do 
capital humano, consegue explicar de forma mais eficaz a variação crosssectional média dos retornos dos portfolios analisados. Isto acontece porque a variável size, ou efeito tamanho, influencia muito o mercado brasileiro .

Em relação ao modelo dinâmico, podemos concluir que, sem dúvida alguma, o poder de explicação do modelo aumenta muito, para qualquer um dos casos analisados.

O modelo parece conseguir captar o efeito dinâmico da economia. Introduzindo a variável size, os modelos têm aumento considerável em seus $\mathrm{R}^{2}$; mas nota-se que esta variável parece ser mais significativa no mercado brasileiro, devido provavelmente às diferenças encontradas nas composições de ações desses mercados.

Finalmente conclui-se que o CAPM condicional de Jagannathan e Wang (1996) para o mercado norte-americano é perfeitamente aplicável ao mercado brasileiro. Os resultados encontrados acima para os mercados nos permitem diferenciar e também identificar algumas características próprias de cada um deles. Isto se torna importante ferramenta para o investidor potencial desses países, já que são encontradas diferenças no comportamento dos mercados analisados.

\section{Artigo recebido em 26.07.2004. Aprovado em 13.09.2004.}

\section{Referências Bibliográficas}

Black, F. (1972, July).

Capital market equilibrium with restricted borrowing. Journal of Business, 45(3), 444-455.

Black, F.,

Jensen, M. C., \&

Scholes, M. (1972).

The capital asset pricing model: some empirical tests. In M. C. Jensen (Ed.), Studies in the theory of capital markets (pp.79-121). New York: Praeger.
Bodurtha, J. N., Jr., \&

Mark, N. C. (1991, September).

Testing the CAPM with time-varying risks and returns. Journal of Finance, 46(4), 1485-1505.

Bonono, M., \&

Garcia, R. (2002).

Estimando e testando o CAPM condicional com efeitos ARCH para o mercado acionário brasileiro. In $\mathrm{M}$. Bonomo (Ed.). Finanças aplicadas ao Brasil (pp.41-52). Rio de Janeiro: Editora FGV. 
Fama, E. F., \&

French, K. R. (1992, June).

The cross-section of expected stock returns. Journal of Finance, 47(2), 427-466.

Fama, E. F., \&

French, K. R. (1993, February).

Common risk factors in the returns on bonds and stocks. Journal of Financial Economics, 33(1), 3-56.

Fama, E. F., \&

French, K. R. (1988, October).

Dividend yields and expected stock returns. Journal of Financial Economics, 22(1), 3-25.

Fama, E. F, \&

MacBeth, J. D. (1973, May/June).

Risk, return and equilibrium: Empirical tests. Journal of Political Economy, 81(3), 607-636.

Ferson, W. E. \&

Harvey, C. R. (1999, April).

The variation of economic risk premiums. Journal of Political Economy, 99(2), 385-415.

Haugen, R. A. (1986).

Modern Investment Theory (3rd ed.). New Jersey: Prentice - Hall.

Jagannathan, R., \&

Wang Z. (1996, March).

The Conditional CAPM and the CrossSection of Expected Returns. Journal of Finance, 51(Issue 1), 3-53.
Lintner, J. (1965, February).

The valuation of risk assets and the selection of risk investments in stock portfolio and capital budgets. Review of Economics and Statistics, 47(1), 1337.

Mayers, D. (1972).

Nonmarketable assets and capital market equilibrium under uncertainty. In M. C. Jensen (Ed.), Studies in the theory of capital markets (pp.223248). New York: Praeger.

Merton, R. C. (1973, September).

An intertemporal capital asset pricing model. Econometrica, 41(5), 867-887.

Mossin, J. (1966, October).

Equilibrium in a capital asset market. Econometrica, 34(4), 768-783.

Ribenboim, G. (2002).

Testes de versões do modelo CAPM no Brasil. In M. Bonomo (Ed.). Finanças Aplicadas ao Brasil. (pp. 17-40). Rio de Janeiro: FGV Editora.

Sharpe, W. F. (1964, September).

Capital asset prices: A theory of market equilibrium under conditions of risk. Journal of Finance, 19(3), 425442.

Treynor, J. L. (1961).

Toward a theory of the market value of risky assets. Manuscrito não publicado. 